\title{
Environmentally friendly technology for the modification of lubricants with graphene nanostructures
}

\author{
Zaman A A Alhilo ${ }^{*}$, Gaukhar Zhumagalieva ${ }^{2}$, and Tatiana Pasko ${ }^{3}$ \\ ${ }^{1}$ Post-graduate student from the Republic of Iraq, Tambov State Technical University, 106 \\ Sovetskaya Street, 392000, Tambov, Russian Federation \\ ${ }^{2}$ Zhangir Khan West Kazakhstan Agrarian-Technical University, Zhangir Khan Str., 51, Uralsk, \\ 090009, Republic of Kazakhstan \\ ${ }^{3}$ Department of Technology and Methods of Nanoproducts Manufacturing, Tambov State Technical \\ University, 106 Sovetskaya Street, 392000, Tambov, Russian Federation
}

\begin{abstract}
Nanomaterials, and in particular graphene, significantly increase the basic tribological characteristics of lubricants. One of the most effective ways to quickly use laboratory results on an industrial scale is to modify traditional lubricants with graphene concentrates. The research results showed that in order to solve this problem successfully, it is necessary to create graphene concentrate and distribute it evenly over the total volume of the lubricant. It is necessary that the concentrate be prepared on the same basis as the lubricant. In the present work, previous studies on the mechanical activation and exfoliation of graphite in a core drum mill, liquid-phase shear exfoliation in a rotary apparatus with moving blades, and the homogenization of graphene nanostructures in lubricants using a rotary mixer are combined. Changes were made to the design of the main components, taking into account the use of this technology in the industry. The presented technology is environmentally friendly, since only oil and graphite are used and there are no harmful wastes that must be disposed of.
\end{abstract}

\section{Introduction}

Currently, lubricants have high requirements regarding antifriction and anti-wear characteristics in friction units of many mechanisms and machines operating at variable and elevated speeds, pressures and under various temperature conditions [1]. The formation of composite lubricants with desired properties has become the main direction of development of modern tribology. The anisotropic nanofriction force on $\mathrm{MoS}_{2}$ as a function of thickness was studied by a calibrated atomic force microscopy (AFM) in ambient air. The observed anisotropic nanofriction on $\mathrm{MoS}_{2}$ with a thickness of $4.18 \mathrm{~nm}$ was attributed to the combination of the lattice orientation and puckering effect. The anisotropic nanofriction on

*Corresponding author: tpasko@yandex.ru 
$\mathrm{MoS}_{2}$ with a thickness of $1.49 \mathrm{~nm}$ was mainly due to the puckering effect dominating the nanofriction. As the thickness of $\mathrm{MoS}_{2}$ increased, the magnitude of nanofriction decreased, and the anisotropy ratio of nanofriction increased. The structural anisotropy coupling with thickness plays an important role in the anisotropic nanofriction on $\mathrm{MoS}_{2}$. This study could provide the clue for the design of $\mathrm{MoS}_{2}$ and other two-dimensional materials in lubrication applications.

Graphite is traditionally used as an antifriction material [2]. A new explosion of interest in materials that can be obtained from graphite occurred after graphene was obtained and its unique physical and mechanical characteristics were determined. It was experimentally established that one layer of graphene grown on steel withstands 6400 slip cycles, while low-layer graphene (consisting of 3-4 layers) withstands 47,000 cycles. In addition, it was shown that these graphene layers completely stop wear, despite the difficult sliding conditions, including high contact pressures $(\approx 0.5 \mathrm{GPa})$, which are usually observed in small-scale trials [3]. The experimental results demonstrate that the addition of $0.4 \mathrm{wt} . \%$ Graphene nanofiller to lithium lubricants gives good antifriction properties and wear resistance indicators [4]. Theories of friction and graphite wear were analyzed in [5]. The combined action of graphite and graphene in grease can reduce the friction coefficient by 2.2-2.5 times [6]. Adding graphene concentrate to a standard lubricant is one of the promising ways to increase the tribological characteristics of lubricants.

Earlier, we analyzed the effect of carbon nanomaterials on the tribological characteristics of lubricants [7]. In spite of good development and progress in lubricants, billions US dollars are being lost in many industries and motor vehicles globally due to mechanisms frictions. Therefore, there is a great need to develop more efficient and effective lubricants. The review paper considers the application of carbon nanostructures, such as carbon nanotubes and graphene, as nano-additives to lubricants in order to improve their tribological characteristics i.e. to decrease friction and wear. Some important aspects of nanoparticles in modifying lubricants are size, form, structure, surface functionalization, concentration, physical and chemical properties of surfaces, which are outlined in this article. It was observed that the adding of nano-additives to lubricants; even at low concentrations; decreased the friction and wear coefficients considerably. Moreover, carbon nanotubes and graphene are environmentally friendly and safe. Thus, all these factors allow positive assessment of the prospects of using the above mentioned nanomaterials to modify lubricants.

The review [5] compares the classical theory of friction and wear with the modern one. The advantages and disadvantages of each of these theories are analyzed. In addition, the graphology tribology, the prospects of its use are considered and shown.

In recent decades, much attention has been paid to studies of the effect of nanoscale fillers and, in particular, graphene nanostructures on the performance of lubricants. Twodimension graphene-like covalent-organic frameworks (GCF) nano-sheets were successfully fabricated to evaluate the tribological behavior as lubricant additive in comparison to GO and rGO in PAO 10 oil [8]. The mechanical strength of covalent bond structure in-plane ensures carrying capacity and durability, and the ultrathin layered feature reduces shear stress due to slide effect. Eventually, the excellent reducing friction (up to $53.5 \%$ ) and anti-wear (up to $95.4 \%$ ) were achieved at an ultralow dosage of $0.008 \mathrm{wt} \%$. By contrast, dissociation of $\mathrm{C}-\mathrm{O}$ linkages of $\mathrm{rGO}$ and $\mathrm{GO}$ induced by tribo-stress and triboheat deteriorates its stability and durability.

Graphite oxide (GO) with $\mathrm{N}$ doping is synthesized from the waste carbon source. The GO products obtained at $1200-1400{ }^{\circ} \mathrm{C}$ are utilized as nano-additive in synthetic engine oil [9]. The GO is produced in the form of layered structure with Ca compound incorporation and functional groups. GO addition has improved the friction $(2.8-21.1 \%$ reduction) and 
wear $(8.2-18.5 \%$ reduction in worn volume) properties due to its easy shear force of layered structure.

Natural crystalline graphite of GSM-2 grade (ash content $<0.5 \%$ ) was used in the study [10]. The oxidative intercalation of graphite was carried out using ammonium persulfate (pure for analysis grade) dissolved in sulfuric acid containing 5\% free sulfur trioxide. Intercalated graphite was subjected to cold expansion at $40^{\circ} \mathrm{C}$ for $3 \mathrm{~h}$. The apparent volume of graphite is $270-280 \mathrm{~cm} 3 / \mathrm{g}$ of the initial graphite. In order to prepare a paste to be used for mechanical treatment, carbamide (on the basis of 0.5 mole per 1 mole of sulfuric acid contained in EGC) was added to EGC. The reaction mixture self-heated to $80^{\circ} \mathrm{C}$ and a thick gray-green paste was formed. Glycerol ( $100 \mathrm{~g}$ per $5 \mathrm{~g}$ of the initial graphite) was then added to this paste. The resulting paste was treated in a stainless steel cylinder-shaped container with an internal diameter of $60.0 \mathrm{~mm}$ and a steel rotor $59.3 \mathrm{~mm}$ in diameter rotating inside the container. The net time of treatment was $30 \mathrm{~min}$. The GNPs in the form of an aqueous paste containing $3-5 \%$ of dry substance were obtained.

GNPs are successfully produced in rod drum mills [11, 12] and rotary apparatuses (stator-rotor mixer) [13, 14].

In our opinion, it is better to use graphene nanoparticles (GNP) to modify lubricants than graphene oxide because the technology for producing GNP is environmentally friendly. It should be noted that they are used to modify not only lubricants, but also other materials, such as epoxy $[15,16]$.

It is very important that using the method of liquid-phase shear exfoliation it is possible to produce GNP directly in synthetic oils, and on an industrial scale $[17,18]$. In addition, graphene-modified lubricants can operate at low temperatures [19, 20].

This article proposes a technology for the modification of lubricants with graphene nanostructures obtained by shear exfoliation of graphite, which can be implemented on an industrial scale.

\section{Technology for the production of graphene structures and the modification of lubricants}

Figure 1 shows a scheme of the modification of lubricants by graphene nanostructures.

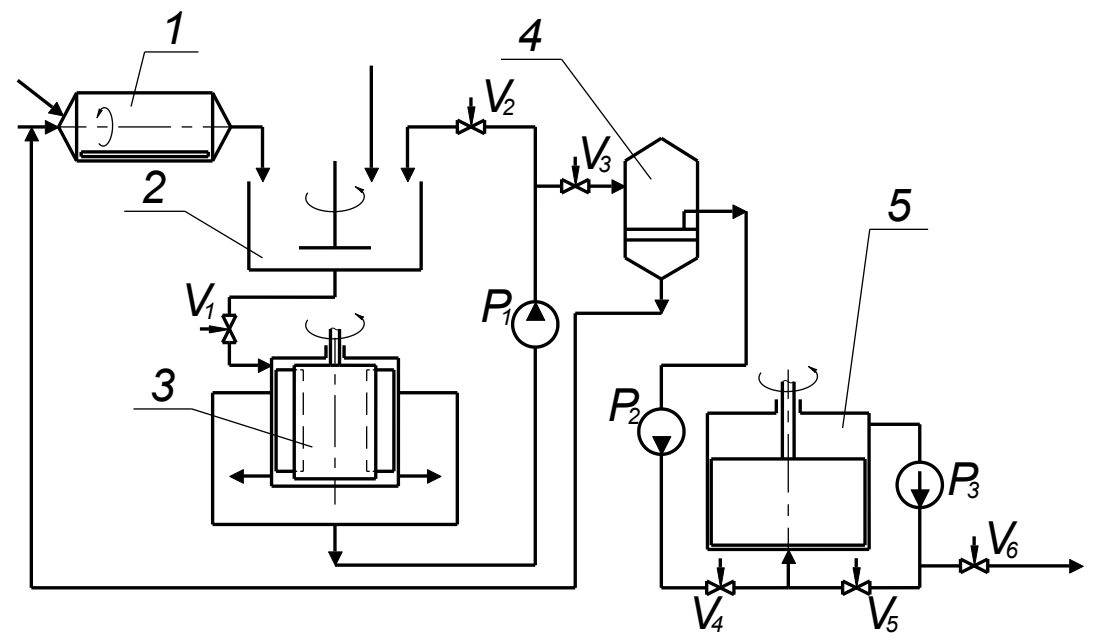

Fig. 1. Functional diagram of the production of graphene nanostructures and the modification of lubricants: 1 - rod drum mill; 2 - stirred tank; 3 - rotary apparatus (exfoliator); 4 - filter; 5 - rotary mixer; 6 - rotary homogenizer; $P_{1}, P_{2}, P_{3}$ - pumps; $V_{1}, V_{2}, V_{3}, V_{4}, V_{5}, V_{6}-$ valves. 
This technology is implemented as follows. Pre-prepare a mixture of synthetic oil and crystalline graphite powder and load them into a rod drum mill 1 . Mass concentration of graphite is from 10 to $20 \%$. In the mill, graphite is mechanically activated and partially exfoliated, i.e. stratification of graphite particles into graphene nanostructures. The term "graphene nanostructures" should be understood as a mixture of particles consisting of a different number of graphene layers. Graphene nanostructures contain both single-layer graphene and low-layer and even multi-layer graphene. However, all particles have at least one of the sizes less than 100 nanometers. The duration of processing the mixture in the mill, depending on the desired result and the type of synthetic oil, can vary from 1 to 5 hours. A little later we will discuss the features of processing the mixture in the mill. After mechanical activation is complete, the mixture is reloaded into a container 2 and either pure synthetic oil or clarified suspensions from the filter 4 are added. After adding oil, the concentration of graphite in the suspension should be $1-2 \%$. Open the crane 1 and turn on the rotary drive of the exfoliator 3 . The design of the exfoliator is similar to the one used in the work. The exfoliator consists of a cylindrical stator inside which a rotor with movable blades is installed. The stator is closed by covers at the top and bottom. The suspension is fed into the hole, which is located at the top of the stator, and exits through the hole at the bottom of the stator. Under the action of centrifugal forces, the movable rotor blades are pressed against the inner surface of the stator and constantly slide along this surface. Graphite particles and graphene nanoparticles that are in suspension are pressed against the inner surface of the stator because their density is greater than the density of synthetic oil. Thus, the particles fall between the stationary stator and the moving blade and dissolve under the action of shear forces, i.e. two particles are formed from one particle. This is the mechanism of shear exfoliation in a rotary apparatus with moving blades. From the exfoliator 3, the suspension is pumped into the container 2 and then fed back to the exfoliator. Thus, the exfoliator operates in a cyclic mode. Processing the suspension is from 1 to 5 hours. Exfoliation lasts from 1 to 5 hours. At the end of exfoliation, close the valve 2 and open the valve 3 . The suspension is fed to the filter. The liquid part of the suspension, which contains a certain amount of low-graphene graphene, is returned to the beginning of the process and is used either to prepare the initial mixture for the mill, or to dilute the mixture after processing in the mill in a container with a mixer 2 . The precipitate from the filter 4 is fed into the mixer 5 , which is also fed lubricant to be modified. From the mixer 5 , a mixture of lubricant and graphene nanostructures is supplied to the rotary homogenizer 6 by a pump $P_{2}$. If the valves are closed and the valve is open, the mixture is processed in the homogenizer in a closed cycle for from 0.5 to 1 hour, i.e. the mixture goes through the garlic homogenizer many times. In a homogenizer, graphene nanostructures are not only uniformly distributed over the entire volume of the lubricant, but also exfoliate due to the high velocity gradient in a very thin layer of lubricant. After processing the mixture in the homogenizer, close the valve $V_{5}$, open the valve $V_{6}$ and unload the finished product lubricant modified with graphene nanostructures.

\section{Discussion}

First of all, it should be noted that the proposed technology is an almost continuous production. Two nodes are limiting by the processing time: a mill; rotary apparatus. These nodes can work simultaneously and the processing time of the material in each of them is the same from 1 to 2 hours. Thus, the period of time when processing of the material does not occur is the time the material moves from the mill 1 to the tank 2.

Now consider the features of processing a mixture of graphite with synthetic oils in the mill 1. Figure 2 shows the cross section of a drum mill. Grinding rods 2 are located inside the drum 1 , which are interconnected by a flexible connection 3 and rigidly fixed relative to 
the base of the mill 4 . Since the drum 1 rotates at a speed greater than critical, that is, the centrifugal forces that act on the mixture are greater than the forces of gravity, the mixture forms a thin ring 5, which rotates together with the drum 1 . The particles of graphite together fall into the contact zone between the rotating shell of the drum and the fixed rods. Shear forces begin to act on these particles and the particles exfoliate. Since these effects occur repeatedly, graphite particles gradually turn into particles of multilayer graphene, then into particles of few-layer graphene, and ultimately into single-layer graphene.

The results of our experiments showed that the preliminary mechanical activation of a mixture of graphite and oil in a core drum mill significantly increases the intensity of subsequent exfoliation, therefore it is not advisable to process the mixture in a drum mill for more than 2 hours, since a high concentration of graphene nanostructures in the oil can be obtained much faster in a rotary apparatus. It should be noted that with an increase in the concentration of graphene nanostructures in oil, its viscosity increases [19] and therefore the productivity of the rotor apparatus decreases.

Significant changes have also been made to the design of the rotary mixer compared to the design that we used earlier. The rotor height was increased, and therefore the homogenization of a mixture of lubricants with graphene nanostructures and exfoliation of graphene nanostructures is carried out not only when moving along a spiral path in the horizontal plane, but also when moving along a helical line in the cylindrical contact zone of the stator and rotor.

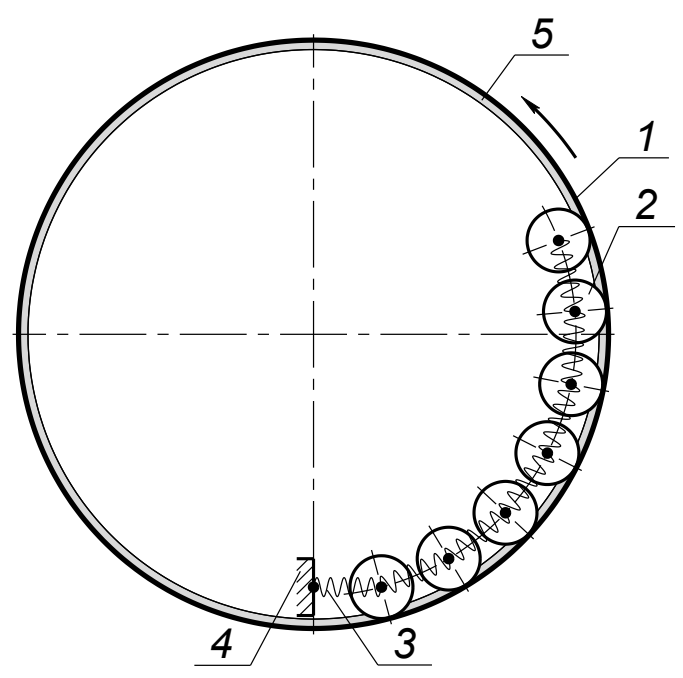

Fig. 2. Cross section of a drum mill: 1 - drum; 2 - rod; 3 - flexible connection; 4 - mill base; 5 mixture of graphite and oil.

\section{Conclusion}

In the present work, previous studies on the mechanical activation and exfoliation of graphite in a core drum mill, liquid-phase shear exfoliation in a rotary apparatus with moving blades, and the homogenization of graphene nanostructures in lubricants using a rotary mixer are combined. Changes were made to the design of the main components, taking into account the use of this technology in the industry. The presented technology is environmentally friendly, since only oil and graphite are used and there are no harmful wastes that must be disposed of. 
The reported study was funded by RFBR, project number 19-38-90159.

\section{References}

1. X. Cao, X. Gan, H. Lang, K. Yu, Sh. Ding, Y. Peng, W. Yi, Tribol. Int. 134, 308-316 (2019)

2. M. Schmitt, S. Bistac, Kh. Jradi, J. Phys. Conf. Ser. 61(1), 1032-1036 (2007)

3. D. Berman, S.A. Deshmukh, Adv. Funct. Mater. 24(42), 6640-6646 (2014)

4. A.A. Gomaa, M.I. Khashaba, J. Multidiscip. Eng. Sci. Technol. 3(8), 5459-5465 (2016)

5. S. Thakur, K.K. Thakur, H. Singh, AGU Int. J. Eng. Technol. 4, 62-69 (2017)

6. D.A.Y. Al-Saadi, V.F. Pershin, B.N. Salimov, S.A. Montaev, J. Frict. Wear 38(5), 355358 (2017)

7. I. Ali, Al A. Basheer, A. Kucherova, N. Memetov, T. Pasko, K. Ovchinnikov, V. Pershin, D. Kuznetsov, E. Galunin, V. Grachev, A. Tkachev, J Mol. Liq. 279 (1), 251266 (2019)

8. P. Wen, Y. Lei, W. Li, M. Fan, Tribol. Int. 143, 106051 (2020)

9. B. Sivakumar, N. Ranjan, S. Ramaprabhu, M. Kamaraj, Tribol. Int. 142, 105990 (2020)

10. A.V. Melezhik, V.F. Pershin, N.R. Memetov, A.G. Tkachev, Nanotechnol. Russ. 7-8, 421-429 (2016)

11. G. Zhumagalieva, V. Pershin, A. Tkachev, A. Vorobiev, A. Pasko, E. Galunin, AIP Conf. Proc. 2041, 020010 (2018)

12. V.F. Pershin, G.B. Zhumagaliyeva, A.G. Tkachev, A.A. Pasko, A.M. Vorobyev, IOP Conf. Ser-Mat. Sci. 693(1), 012035 (2019)

13. K.A. Al-Shiblawi, V.F. Pershin, V.P. Jarcev, T.V. Pasko, AIP Conf. Proc. 2041, 020015 (2018)

14. V. Pershin, K.A. Al-Shiblawi, A.M.R. Al-Mashhadani, A. Pasko, D. Melekhin, IOP Conf. Ser-Mat. Sci. 693(1), 012046 (2019)

15. K.A. Al-Shiblawi, V.F. Pershin, V.P. Yartsev, Mater. Sci. Forum 2, 68-78 (2018)

16. K.A. Al-Shiblawi, V.F. Pershin, V.P. Yartsev, Mater. Sci. Forum 4, 42-53 (2018)

17. V.F. Pershin, M.N. Krasnyanskiy, Z.A.A. Alhilo, A.M.R. Al-Mashhadani, A.A. Baranov, A.A. Osipov, IOP Conf. Ser.-Mat. Sci. 693(1), 012023 (2019)

18. V.F. Pershin, K.A. Ovchinnikov, A.A. Alsilo, R.A. Stolyarov, N.R. Memetov, Nanotechnol. Russ. 13(5-6), 344-348 (2018)

19. V. Pershin, K. Ovchinnikov, Z. Al-Hilo, N. Memetov, A. Tkachev, E. Galunin, AIP Conf. Proc. 2041, 020016 (2018)

20. V. Pershin, G. Zhumagalieva, A. Tkachev, A. Pasko, N. Memetov, AIP Conf. Proc. 2101, 020011 (2019) 